Man and Nature

L'homme et la nature

\title{
Lait Républicain : Les Conventionnels et la Fonction Sociale de la Maternité
}

\section{Michel Grenon}

Volume 10, 1991

URI : https://id.erudit.org/iderudit/1012621ar

DOI : https://doi.org/10.7202/1012621ar

Aller au sommaire du numéro

Éditeur(s)

Canadian Society for Eighteenth-Century Studies / Société canadienne d'étude du dix-huitième siècle

ISSN

0824-3298 (imprimé)

1927-8810 (numérique)

Découvrir la revue

Citer cet article

Grenon, M. (1991). Lait Républicain : Les Conventionnels et la Fonction Sociale de la Maternité. Man and Nature / L'homme et la nature, 10, 39-46.

https://doi.org/10.7202/1012621ar

Copyright (C Canadian Society for Eighteenth-Century Studies / Sociéte canadienne d'étude du dix-huitième siècle, 1991
Ce document est protégé par la loi sur le droit d'auteur. L’utilisation des services d'Érudit (y compris la reproduction) est assujettie à sa politique d'utilisation que vous pouvez consulter en ligne.

https://apropos.erudit.org/fr/usagers/politique-dutilisation/ 


\section{Lait Républicain: Les Conventionnels et la Fonction Sociale de la Maternité}

'Un enfant, en ouvrant les yeux, doit voir la patrie, et jusqu'à la mort ne doit plus voir qu'elle. Tout vrai républicain suça avec le lait de sa mère l'amour de sa patrie: c'est-à-dire, des lois et de la liberté.' ${ }^{1}$ Ces aphorismes éloquents, qu'on peut lire au premier paragraphe du chapitre IV ('Éducation') des Considérations sur le gouvernement de Pologne de Rousseau, seront souvent évoqués durant la Révolution française. Ils résonnent dans l'enceinte de la Convention nationale, ils émaillent plus d'un document législatif. 'On a tiré des ouvrages des philosophes les principes des gouvernements et de la politique. Il est temps d'en tirer ceux de la morale universelle; et les moeurs, les moeurs indifférentes au despotisme, mais nécessaires à la liberté, toucheront à leur regénération. Il est surtout nécessaire de pénétrer tous les coeurs de cet amour brûlant de la patrie, qui est la première vertu d'un peuple libre. Que l'enfant, en ouvrant les yeux, voie la patrie, et que jusqu'à la mort il ne voie plus qu'elle!' ${ }^{2}$ Ainsi s'exprime Arbogast, député du Département du BasRhin et membre du comité d'instruction publique de la Convention nationale.

L'éducation et son orientation dans la société nouvelle constituent l'un des débats - l'un des enjeux - de la Révolution. Ce débat, commencé avant la chute de l'Ancien Régime, traverse les États généraux, prend de l'ampleur sous la Législative et rebondit sous la Convention. Si l'inspiration, si certains postulats sont empruntés à Rousseau - 'O Rousseau! ô mon maître,' s' exclame Michel-Edme Petit, député de l'Aisne, en décembre $1792^{3}$ - d'anciennes et honorables notions sont également réquisitionnées et, chemin faisant, rajeunies: patrie, corps politique, nature ... La figure de la mère nourricière y occupe une position importante. Le même Michel-Edme Petit déclare encore: '...l'éducation en général (...) doit aller chercher l'homme dans l'embryon de l'espèce; et ce n'est pas encore assez, les pères et les mères surtout, doivent d'abord fixer son attention. ${ }^{4}$ Avant même de donner au peuple des écoles primaires, ajoute-t-il, la Convention doit rappeler les mères 'au devoir de donner leur lait à leurs enfants. ${ }^{5}$ 
La France devra attendre la réaction thermidorienne avant de voir s'apaiser le débat sur l'orientation de l'éducation nationale. Dès les débuts de la Révolution, pourtant, on avait proclamé l'urgence d'organiser un nouveau régime scolaire. De nombreux facteurs expliquent cet échec relatif; les analyser ici nous entraînerait trop loin. Notons pourtant que les députés furent incapables de s'entendre sur les principes mêmes sur lesquels faire reposer ce régime. $S^{\prime}$ il est un point, cependant, sur lequel règne un certain consensus, $c^{\prime}$ est la place qui revient aux mères dans la formation des nouveaux citoyens. Au printemps 1793, alors que la jeune République recule devant l'ennemi extérieur, que la guerre civile éclate et que la Convention nationale elle-même est gravement éprouvée par l'affrontement des Girondins et des Montagnards, le député P.C.F. Dupont propose à ses collègues un projet de décret 'Sur les bases de l'éducation nationale' qui comporte les articles suivants:

ART. 25. (Les institutrices) feront des instructions sur l'économie rurale domestique, et sur les devoirs à remplir par les mères de famille.

ART. 26. On honorera spécialement celles qui allaitent leurs enfants.

ART. 27. Il sera fait une instruction ou adresse à toutes les mères de famille de la République pour leur rappeler cet important devoir, et les dangers auxquels elles s'exposent en négligeant de le remplir. ${ }^{6}$

Dans la République, les mères ont le devoir d'allaiter leurs enfants. Ce devoir, cette fonction découle de la place des femmes dans la société; elle est dictée par la nature. Dupont a inscrit ce postulat au début de son projet de décret:

ART. 8. L'éducation devant prendre la nature pour modèle, il sera fait une distinction des arts et des métiers qui devront être exercés par les femmes, et de ceuxà exercer par les hommes.

ART. 9. Nul homme ne pourra exercer un métier de femme, sans perdre le droit de citoyen.

ART. 10. L'homme qui épouserait une femme qui exercerait le métier des hommes perdra son droit de citoyen. ${ }^{7}$

La nature distribue aux hommes et aux femmes leurs fonctions spécifiques; le bon citoyen, la bonne citoyenne, acceptent ses volontés. Du- 
pont développe encore cet argument dans un texte qui accompagne son projet de décret: Bases de l'éducation publique, ou l'art de former les hommes; par P.-C.-Fr. Dupont, des Hautes-Pyrénées. 'La nature, déclare-t-il, immense dans tous ses rapports, est infiniment féconde dans ses variétés; elle ne produit pas deux êtres parfaitement semblables. La différence des organes fait la différence des goûts, des inclinations et des penchants dans les individus, comme dans les différents sexes.' La figure $d^{\prime} H e r c u l e$ jouit alors d'une grande popularité parmi les révolutionnaires: mais Hercule filant la laine aux pieds de la reine Omphale, précise-t-il, est une monstruosité. Il poursuit: 'Je pose donc, comme premier principe, qu'il y a certains arts qui ne peuvent être exercés que par les hommes, comme d'autres sont du domaine des femmes. Un bon plan d'éducation doit les distinguer. Le sabre et l'épée doivent être aussi étrangers à la femme que la quenouille à l'homme.' Et il ajoute: 'Quand je vois l'aiguille entre les mains d'un homme vigoureux, je vois un voleur qui s'empare de l'industrie d'autrui, ou un homme qui allaite un enfant.'

L'allaitement appartient à la femme et dans la République, cette fonction confère aux mères nourrices un rôle capital. Dans ses Idées sur l'éducation nationale, Alexandre Deleyre, député de la Gironde (mais il ne frayait pas avec les 'Girondins'), veut que la République honore tout spécialement Rousseau pour avoir su reconnaître l'importance de l'allaitement. 'Rousseau, dit-il, certainement a plus fait pour les enfants qu'Anaxagore. Il leur a rendu, pour ainsi dire, le lait de leurs mères...' Une fête annuelle devrait lui être consacrée, qu'il propose d'appeler la Jean-Jacques ou fête des enfants. 'Ils iraient tous habillés, les garçons à la Jean-Jacques, les filles en jaquette ou robe blanche, un peu courte, sur deux files, à côté de leurs mères qui formant une bande au milieu de ces deux, tiendraient par la main leurs plus petits enfants des deux sexes qui auraient besoin d'être menés.' Il ajoute, à propos des mères: 'Celles qui seraient nourrices, porteraient les leurs à cette fête.' Deleyre souhaite d'ailleurs que le spectacle de l'allaitement soit inscrit au programme scolaire. Les villages les plus peuplés, dit-il, devraient prévoir un lieu une salle, 'ou même une grange' - où les nourrices se réuniraient une fois la semaine, afin que les élèves puissent contempler cette 'première éducation de la vie humaine' et ne perdent pas l'habitude de 'voir des enfants à la mamelle. ${ }^{\prime 9}$

Dans la mouvance montagnarde, l'allaitement possède un sens explicitement politique. Michel Lepeletier, ci-devant marquis et pour l'heure député de l'Yonne à la Convention, assassiné (janvier 1793) par un ancien garde du corps du roi pour avoir voté la mort de Louis XVI, laissait dans ses papiers un Plan d'éducation nationale, dont Robespierre lui-même fit lecture à la Convention nationale. Lepeletier veut que tous les enfants soient élevés aux dépens de la République dès l'âge de cinq 
ans, jusqu'à l'âge de douze ans pour les garçons, de onze pour les filles; dans cette école obligatoire, ils recevront tous 'même nourriture, mêmes vêtements, même instruction, même soins.' Avant l'âge de cinq ans cependant, la République ne peut faire mieux que de laisser les enfants aux soins des mères - 'c'est le voeu, c'est le besoin de la nature,' précise-t-il. Cela dit, la loi peut déjà influencer ces vies naissantes, notamment en intéressant efficacement les mères à allaiter leurs enfants. L'article 4 du projet de décret qui accompagne son texte stipule qu'une mère, conduisant son enfant à l'école pour la première fois, recevra une récompense monétaire. L'article précise qu'aucune mère ne pourra refuser cette récompense mais qu'en même temps, elle n'y aura droit que si la municipalité atteste qu'elle a bien allaité son enfant. L'article 5 ordonne que des manuels soient rédigés à l'intention des mères, pour les conseiller durant la grossesse, l'allaitement, le sevrage et la croissance des enfants jusqu'à leur cinquième année. ${ }^{10}$ Le 29 juillet 1793, Robespierre fait lecture, en son propre nom, d'un nouveau projet de décret sur 'l'éducation publique,' qui reprend mot pour mot les articles 4 et $5 \mathrm{du}$ projet de décret Lepeletier. ${ }^{11}$

Les Montagnards domineront la Convention nationale, grosso modo, de janvier 1793 à l'été 1794. Période fertileen crises de toutes sortes, et jusque dans les rangs des Montagnards eux-mêmes: l'affrontement meurtrier des dantonistes et des robespierristes en est un exemplespectaculaire. Un certain idéal politique et social est associé à ce chapitre de l'histoire de la Convention: la Terreur en est l'expression extrême. L'expression pédagogique de cet idéal se condense en cette formule lapidaire de Lepeletier: 'Créer un nouveau peuple.' Dans cette entreprise, la mère nourrice est toujours au premier rang: elle, et son lait, sont consubstantiels à la patrie. En juillet 1793, après avoir rappelé que la patrie est la mère commune, Grégoire demande aux mères de la République de remplir 'le devoir sacré' d'allaiter elles-mêmes leurs enfants. ${ }^{12}$ Peu après, le député de Paris Fourcroy, rendant hommage à Lepeletier - nouveau martyr de la liberté - rappelle que les enfants ont 'une première mère: $c^{\prime}$ est la patrie.' Grâce au plan Lepeletier, dit-il, les enfants seront recueillis dans 'le sein de cette mère commune' et nourris 'de sa propre substance. ${ }^{13}$ Thibaudeau, député de la Vienne, qui s'oppose pourtant, avec d'autres, aux modalités draconniennes du plan Lepeletier, déclare: 'J'ai toujours pensé que les enfants étaient une propriété de l'État, et que les parents n'en étaient que les dépositaires; que c'était à l'État à recevoir, pour ainsi dire, l'enfant du sein de sa mère; qu'il devait s'en emparer comme de son bien le plus précieux; qu'il fallait quel'enfant, en ouvrant les yeux, ne vît quela patrie, et que jusqu'à la mort il ne vît plus qu'elle.' Et de rappeler que 'la véritable nourrice est la mère. ${ }^{14}$ Un autre Montagnard, Gabriel Bouquier, député de la Dordogne, présenta son propre plan, à l'automne 1793. C'est au 
cours de ce nouveau débat que Danton s'écrie, selon le Moniteur: 'C'est dans les écoles nationales que l'enfant doit sucer le lait républicain.. ${ }^{15}$ Dans les 'fragments' que Saint-Just rédige vers cette époque, publiés après sa mort sous le titre Institutions républicaines, on lit: 'Les enfants appartiennent à leur mère jusqu'à cinq ans, si elle les a nourris, et à la république ensuite, jusqu'à la mort.' Et encore ceci: 'La mère qui n'a point nourri son enfant a cessé d'être mère aux yeux de la patrie. Elle et son époux doivent se représenter devant le magistrat, pour y répéter leur engagement, ou leur union n'a plus d'effets civils. ${ }^{16}$ Le 22 janvier 1794, à l'occasion de l'ouverture d'un concours pour les manuels de l'éducation élémentaire, Grégoire déclare: 'On n'annonce rien de neuf en disant que l'impossibilité physique ou morale peut seule dispenser une mère d'allaiter son enfant; mais il faut que désormais l'opinion publique flétrisse celles qui, foulant aux pieds un devoir sacré, ne donnent à la patrie un nouvel individu que pour se hâter de l'abandonner à des mains mercenaires. ${ }^{17}$

La chute de Robespierre est suivie de nouvelles manoeuvres dans le domaine de l'éducation. La Convention adoptera finalement une loi générale sur l'enseignement en brumaire an IV (octobre 1795). Les images rousseauistes s'estompent, de même que le grand dessein de regénération: s'estompent également les appels conjugués à la patrie et à la mère, à la République et à son lait, à la nature et aux devoirs féminins.

La politisation, la politique de l'allaitement maternel possédait une référence sociale spécifique: il s'agissait de l'une des plus funestes institutions de l'Ancien Régime, les 'nourrices' rurales à qui les jeunes mères citadines confiaient volontiers leurs nouveaux-nés. ${ }^{18}$ Dans son rapport sur les livres élémentaires cité plus haut, Grégoire déclare: 'La Convention nationale n'entend prononcer qu'avec peine le mot de nourrice. Elle éprouve un sentiment douloureux en apprenant que les registres de l'an dernier du bureau des recommanderesses (sic) présentent encore, pour Paris seulement, près de six mille enfants qui n'ont pas sucé le lait maternel, sans compter un nombre supérieur peut-être, tant de ceux qui ont été portés aux enfants trouvés (sic) que de ceux qui ont été envoyés en nourrice sans passer par l'intermédiaire de l'un ou l'autre de ces dépôts. ${ }^{19}$ Grégoire lui-même estime à la moitié le nombre des enfants qui périssent avant l'âge de huit ans. ${ }^{20}$ Les conventionnels ont d'ailleurs gardé de l'Ancien Régime la conviction que la France manque de bras.

Il n'est pas étonnant qu'en 1793-4, au moment où la guerre semble atteindre une ampleur inouie, les législateurs s'inquiètent du peuplement. Ainsi se légitime la politique de l'allaitement, ce 'maillon faible du processus reproductif. ${ }^{.21}$ L'allaitement maternel, fonction importante dans ce contexte de guerre, de révolution; fonction importante dans cette 
société du dix-huitième siècle, dont l'évolution démographique, enfin encourageante, est mal perçue; fonction importante aussi dans la vieille campagne des élites masculines pour imposer aux femmes de toutes les conditions, mais aussi aux masses rurales et urbaines, certaines normes de comportement social.

Le discours sur l'allaitement, pour sa part, par-delà sa dimension 'symbolique' qui n'est ni de mon propos ni de ma compétence, figure dans un ensemble conceptuel où les conventionnels circulent constamment. Les origines de cet ensemble sont complexes: les classiques de l'Antiquité y font bon ménage avec les auteurs des Lumières, avec Rousseau. ${ }^{22}$ Peu de conventionnels pensent à retoucher l'édifice, à l'adapter aux temps nouveaux; tous y puisent à pleines mains. Dans cet ensemble, on peut concevoir un parcours où se côtoient l'idée de mère, l'idée de patrie, l'idée de nature. La mère incarne la patrie; la patrie procède de la nature; la nature s'incarne dans la mère. Lorsque la mère emmène pour la première fois son enfant à l'école, Lepeletier peut dire que la patrie reçoit l'enfant des mains de la nature. ${ }^{23}$ Par la même occasion, l'amour maternel est fonction de la nature - il est d'ailleurs 'fort comme la nature $; i^{24}$ de son côté, la nature offre à ses enfants la nourriture 'régénératrice' de ses mamelles. Le peintre David, député de Paris et membre du Comité d'instruction publique, chargé par ses collègues de l'organisation d'une grande cérémonie pour commémorer la prise des Tuileries, imagine un parcours en cinq 'stations' (passons par-dessus ce recours à un terme religieux) dont la première célèbrera la Régénération; 'Le rassemblement, écrit-il, se fera sur l'emplacement de la Bastille. Au milieu de ses décombres, on verra s'élever la fontaine de la Régénération, représentée par la nature. De ses fécondes mamelles, qu'elle pressera de ses mains, jaillira, avec abondance, $l^{\prime}$ eau pure et salutaire.... ${ }^{\prime 25}$ Les mamelles de la nature dispensent la régénération; le sein maternel dispense le lait républicain, substance même de la patrie.

Les éléments de cet ensemble ne possèdent pas tous les mêmes propriétés: l'idée de mère a la propriété d'assembler, de relier: outre la patrie et la nature, elle relie la femme et le corps social. ${ }^{26}$ L'allaitement maternel situe la femme, mamelle du corps social; on lui demande de nourrir, $d$ 'inspirer, de réconforter - mais non de combattre, ni de voter.

\section{MICHEL GRENON}

Université du Québec à Montréal 


\section{Notes}

1 J.J. Rousseau, Les considérations sur le gouvernement de Pologne et sur sa réformation projetée (1872).

2 Rapport et projet de décret sur la composition des livres élémentaires destinés à l'instruction publique, présentés à la Convention nationale au nom du Comité d'instruction publique, par L.-F.-A. Arbogast. Reproduit dans J. Guillaume, Procès-verbaux du Comité d'instruction publique de la Convention nationale (Paris, 1894), 1:96.

3 Opinion de Michel-Edme Petit, député du Département de l'Aisne, contre le projet des écoles primaires, présenté par le Comité d'instruction publique de la Convention nationale. Voir Procès-verbaux, I: 177.

4 Ibid, I: 176.

5 Ibid, I: 181.

6 Projet de décret. Sur les bases de l'éducation nationale. Ibid, I: 675.

7 Ibid, I: 674 .

8 Voir Ibid, I: 672-73.

9 Idées sur l'éducation nationale, par Alexandre Deleyre, député du Département de la Gironde. Voir ibid, I: 655.

10 Plan d'éducation nationale de Michel Lepeletier, présenté à la Convention nationale par Maximilien Robespierre, au nom de la Commission d'instruction publique. Voir ibid, 2: 34-54. Le projet de décret qui accompagne ce texte est divisé en plusieurs sections. Les articles dont il est fait mention ici sont tirés de la section intitulée 'De l'éducation nationale,' ibid, 2: 56.

11 Projet de décret sur l'éducation publique, par le citoyen Robespierre, lu dans la séance du 29 juillet 1793. Voir ibid, 2: 164.

12 Discours du citoyen Grégoire, député du Département de Loir-et-Cher, sur l'éducation commune, prononcéà la séance du 30 juillet. Voir ibid, 2: 173-78.

13 Opinion de Fourcroy, député du Département de Paris, sur le projet d'éducation nationale de Michel Lepeletier, prononcé à la séance du 30 juillet. Voir ibid, 2: 173-78.

14 Discours sur l'éducation publique, par A.-C. Thibaudeau, dans la séance du premier août 1793. Voir ibid, 2: 199 et 204.

15 Le compte-rendu du Moniteur est reproduit dans ibid, 3: 151.

16 L. Saint-Just, Institutions républicaines. Sixième fragment, 'Sur l'Éducation.'

17 Rapport sur l'ouverture d'un concours pour les livres elémentaires de la première éducation, séance du 3 pluviôse, l'an second de la République une et indivisible. Voir ibid, 3: 366 .

18 Bonne introduction dans P. Goubert et D. Roche, Les Français et l'Ancien Régime. Tome 2: Culture et société (Paris, 1984). Voir aussi N. Senior, 'Aspects of Infant Feeding in Eighteenth-Century France,' Eighteenth-Century Studies (1983): 367-88.

19 Le Bureau des recommanderesses, l'Hôpital des enfants trouvés agissaient comme relais vers les nourrices villageoises.

20 Voir Procés-verbaux, 3: 366.

21 N.-C. Mathieu, L'arraisonnement des femmes (Paris, 1985), p. 90. 
22 Voir, entre autres ouvrages récents, C. Mossé, L'Antiquité dans la Révolution française (Paris, 1989).

23 Lepeletier, Plan d'éducation nationale, dans Procès-verbaux, 2: 38.

24 M.-E. Petit, Discours, dans ibid, 3: 418.

25 Les représentants boivent d'une coupe commune, chaque libation étant saluée par un salve d'artillerie. Rapport et décret sur la fête de la réunion républicaine du 10 août, présentée au nom du Comité d'instruction publique par David, député du Département de Paris. Voir ibid, 2: 73 et 263. Voir aussi C. Hould, dir., L'image de la Révolution française (Québec, 1989), p.378.

26 Grégoire, Rapport sur l'ouverture d'un concours, dans Procès-verbaux, 3: 364. 